\title{
Feasibility of multi-parametric magnetic resonance imaging in detection and local staging of prostatic carcinoma
}

Abul-fotouh Ahmed ${ }^{1 *}$, Hesham El-Helaly ${ }^{2}$, Sayed Elawady ${ }^{1}$, Tarek Elzayat ${ }^{3}$, Gamal Abdelhamid ${ }^{4}$, Mohammed M. Agha' and Ahmed Fahim ${ }^{1}$

\begin{abstract}
Background: The objective of this study was to assess the feasibility of multi-parametric magnetic resonance imaging (mpMRl) in detection, localization and local staging of prostate cancer (Pca).

Methods: The study included 58 patients with Pca who underwent mpMRI before radical prostatectomy (RP) at two university hospitals, during the period June 2014 to April 2018. All prostatectomies were performed on the basis of preoperative transrectal ultrasound-guided prostatic biopsies. For tumor localization, the prostate in each patient was divided into six segmental regions. Biopsy specimens, for each segmental region, were evaluated for the presence of cancer. The diagnostic performance of mpMRI in tumor localization as well as extracapsular extension (ECE) and seminal vesicle (SV) invasion of the tumor was evaluated, by using the histopathological findings of RP specimens as reference standard.
\end{abstract}

Results: The mean age of patients was $63.45 \pm 7.45$ years. Of the total number of 348 segmental regions, tumor was detected in 143 . From them, cancer was detected in 142 regions by mpMRI. The sensitivity and specificity of mpMRI for cancer localization were $99.30 \%$ and $97.56 \%$. On RP specimen, nine cases had ECE and five had SV invasion. All of them were detected preoperatively by mpMRI. The sensitivity and specificity of mpMRI for detection of ECE were $100 \%$ and $97.96 \%$. For detection of SV invasion, the sensitivity and specificity were $100 \%$ and $98.11 \%$.

Conclusions: mpMRI enables localization and staging of cancer prostate with reasonable accuracy. Its combination with ultrasound should be counted on for improvement in efficacy of the prostatic biopsy procedure.

Keywords: Prostatic carcinoma, Multi-parametric magnetic resonance imaging, Transrectal ultrasound, Prostatic biopsy

\section{Background}

Most of prostate cancer ( $\mathrm{PCa}$ ) cases are first found during screening with digital rectal examination (DRE) or serum prostatic-specific antigen (PSA) $[1,2]$. The actual diagnosis of $\mathrm{PCa}$ is based only on prostatic biopsies. In 1989, Hodge et al. introduced transrectal ultrasound (TRUS)-guided biopsy which became the core of the

\footnotetext{
*Correspondence: abulfotouhahmed@yahoo.com

${ }^{1}$ Department of Urology, Faculty of Medicine, Al-Azhar University,

Cairo 11633, Egypt

Full list of author information is available at the end of the article
}

standard care in diagnosis of PCa [3]. However, its role in local staging is limited.

Magnetic resonance imaging (MRI) was introduced in 1990s as a tool to aid the locoregional staging of $\mathrm{PCa}$. This traditional technique is based on anatomical sequences only, and its resolution is allowed for only assessment of T3 disease [4]. The recent advent of multiparametric MRI (mpMRI), based on the anatomic T1and T2-weighted imaging (WI) and functional imaging of alteration caused by neoplastic tissue, expanded the scope of MRI in diagnosis and local staging of PCa $[5,6]$. 
The aim of the present study was to get a better understanding the value of mpMRI in detection, localization, and local staging of $\mathrm{PCa}$, using radical prostatectomy (RP) specimens as reference standard.

\section{Methods}

The study included patients with $\mathrm{PCa}$ who underwent mpMRI before RP during the period from June 2014 to April 2018 at our university hospitals. Patients with incomplete medical record, previous prostatic biopsies within 3 months of mpMRI and those with known diagnosis of or non-PCa before mpMRI were excluded. The study protocol was approved by our local ethical committee. The patients' demographic data, clinical characteristics, presenting PSA level, TRUS findings and TRUS biopsy and RP specimen histopathology reports were reviewed and recorded.

All patients underwent mpMRI 3-10 days before undergoing the prostate biopsy. The mpMRI was comprised of T2WI, diffusion WI (DWI), magnetic resonance spectroscopy (MRS) and axial dynamic contrast enhancement (DCE) imaging. Examinations were performed using a 3.0 Tesla scanner, (Achiva, 3T; Philips Healthcare Best, Netherlands) equipped with a pelvic phased array surface coil with the patient in supine position. The guidelines of prostate imaging-reporting and data system (PI-RADS) $\mathrm{v} 2{ }^{\mathrm{TM}}$ [7] were followed in patient preparation, MRI technical specifications, lesion assessment, staging and reporting.

Ultrasound-guided transrectal prostate biopsies were performed under local anesthesia by the guidance of ultrasound [BK-medical, Denmark; supplied with a biplanar transrectal probe $(5-7.5 \mathrm{MHz})]$. A tru-cut 18-gauge needle was used, and a systematic 12-core biopsy was employed.

The prostatic biopsies and RP specimens were examined by an expert histopathologist. For tumor localization, prostate was divided into right and left halves and each half was divided into three segments (base, midgland and apex). Biopsy specimens for each segmental region were evaluated for the presence of cancer.

Data were analyzed using MedCalc statistical software program. Continuous data were expressed as mean \pm standard deviation (SD), median and range. Categorical data were expressed as frequency and percentage. Mann-Whitney $U$ test was used to compare continuous variables, and Chi-square or Fisher exact test was used to compare the categorical variables. The diagnostic accuracy of different diagnostic tools was evaluated by measuring the sensitivity, specificity, positive predictive value (PPV), negative predictive value (NPV) and likelihood ratios. Also the area under the curve (AUC) was measured and presented with its $95 \%$ confidence interval
(CI). An alpha error of $\leq 0.05$ was considered statistically significant.

\section{Results}

Fifty-eight patients were eligible for the study. The age of study subjects ranged from 51 to 78 years (mean $63.45 \pm 7.45$ years). The patients' median presenting the PSA level was $17.60 \mathrm{ng} / \mathrm{mL}$. Tumor staging on DRE was cT1 in $32.76 \%(n=19)$ of cases and cT2 in $67.24 \%$ $(n=39)$. The patients' age, presenting the PSA level and prostate size, is shown in Table 1.

The pathological examination of RP specimen revealed that: $46.55 \%(n=27)$ were pT2b, 39.65\% $(n=23)$ were pT2c, $6.90 \%(n=4)$ were pT3a, and $6.90 \%(n=4)$ were pT3b. No regional lymph node involvement or positive surgical margins were detected in any of the RP specimens. Six cases (10.34\%) had Gleason score: $3+3,21$ (36.21\%): $3+4,29$ (50.00\%): $4+3$ and 2 (3.45\%): $4+5$.

Of the total number of 348 ROIs, cancer was detected in 89 regions (25.57\%) in TRUS-guided biopsies, in 147 regions $(42.24 \%)$ in mpMRI, and in 143 regions $(41.09 \%)$ in RP specimens. Using the histopathological findings of RP specimens as reference standard, the diagnostic performance of preoperative TRUS and mpMRI in tumor localization was as follows: sensitivity (57.34\% and 99.30\%); specificity (96.59\% and 97.56\%); PPV (92.13\% and $96.60 \%$ ); and NPV (76.45\% and 99.50\%) (Table 2).

On RP specimens, ECE was observed in nine (15.52\%) patients. All cases were detected on preoperative mpMRI compared to three on TRUS scan. The diagnostic performance of preoperative TRUS and mpMRI for ECE was as follows: sensitivity (33.33\% and $100 \%)$; specificity $(83.67 \%$ and 97.96\%); PPV (2.04\% and 49.00\%); and NPV (87.23\% and $100 \%$ ) (Table 3 ).

Regarding SV invasion, five (8.62\%) patients had $\mathrm{SV}$ invasion on RP specimen and all were detected by preoperative mpMRI. From those five cases, only two were detected by TRUS scan. The diagnostic performance of preoperative TRUS and mpMRI for SV

Table 1 Patients' age, presenting serum PSA level and prostate size in study subjects

\begin{tabular}{|c|c|c|c|c|c|}
\hline & Minimum & Maximum & Median & Mean & SD \\
\hline Age (years) & 51.00 & 74.00 & 64.00 & 63.77 & 7.21 \\
\hline $\begin{array}{l}\text { Total serum PSA (ng/ } \\
\quad \mathrm{mL})\end{array}$ & 2.60 & 170.00 & 17.40 & 22.55 & 24.27 \\
\hline $\begin{array}{l}\text { Prostate size by TRUS } \\
(\mathrm{cc})\end{array}$ & 20.00 & 194.00 & 50.00 & 58.69 & 38.60 \\
\hline $\begin{array}{l}\text { Prostate size by MRI } \\
\quad(c c)\end{array}$ & 17.00 & 190.00 & 50.00 & 58.57 & 37.90 \\
\hline
\end{tabular}

MRI magnetic resonance imaging, PSA prostatic-specific antigen, TRUS transrectal ultrasonography 
Table 2 Diagnostic performance of preoperative TRUS and mpMRI for localization of prostate cancer in the 348 segmental regions in the studied 58 patients

\begin{tabular}{|c|c|c|c|c|c|c|}
\hline & TRUS & & mpMRI & & Histc & ology \\
\hline \multicolumn{7}{|c|}{ Cancer site (number) } \\
\hline Right base & \multicolumn{2}{|l|}{40} & \multicolumn{2}{|l|}{49} & \multicolumn{2}{|l|}{47} \\
\hline Right mid-zonal & \multicolumn{2}{|l|}{31} & \multicolumn{2}{|l|}{43} & \multicolumn{2}{|l|}{43} \\
\hline Right apex & \multicolumn{2}{|l|}{1} & \multicolumn{2}{|l|}{6} & \multicolumn{2}{|l|}{4} \\
\hline Left base & \multicolumn{2}{|l|}{12} & \multicolumn{2}{|l|}{27} & \multicolumn{2}{|l|}{27} \\
\hline Left mid-zonal & \multicolumn{2}{|l|}{7} & \multicolumn{2}{|l|}{18} & \multicolumn{2}{|l|}{18} \\
\hline Left apex & \multicolumn{2}{|l|}{0} & \multicolumn{2}{|l|}{4} & \multicolumn{2}{|l|}{4} \\
\hline \multirow[t]{4}{*}{ Total } & 89 & & 147 & & 143 & \\
\hline & Test + ve & Test - ve & Test + ve & Test - ve & & \\
\hline & 82 & 61 & 142 & 1 & 143 & True + ve \\
\hline & 7 & 198 & 5 & 200 & 205 & True - ve \\
\hline Sensitivity (\%) & \multicolumn{2}{|l|}{57.34 (95\% Cl 48.81-65.57) } & \multicolumn{2}{|l|}{99.30 (95\% Cl 96.17-99.98) } & & \\
\hline Specificity (\%) & \multicolumn{2}{|l|}{96.59 (95\% Cl 93.09-98.62) } & \multicolumn{2}{|l|}{$97.56(95 \%$ Cl 94.40-99.20) } & & \\
\hline Positive PV (\%) & \multicolumn{2}{|l|}{92.13 (95\% Cl 84.46-96.78) } & \multicolumn{2}{|l|}{96.60 (95\% Cl 92.24-98.89) } & & \\
\hline Negative PV (\%) & \multicolumn{2}{|l|}{76.45 (95\% Cl 70.80-81.48) } & \multicolumn{2}{|l|}{99.50 (95\% Cl 97.26-99.99) } & & \\
\hline Positive LHR & \multicolumn{2}{|l|}{16.79 (95\% Cl 08.00-35.26) } & \multicolumn{2}{|l|}{40.71 (95\% Cl 17.13-96.78) } & & \\
\hline Negative LHR & \multicolumn{2}{|l|}{00.44 (95\% Cl 00.36-00.54) } & \multicolumn{2}{|l|}{$00.01(95 \%$ Cl 00.00-00.05) } & & \\
\hline AUC & \multicolumn{2}{|l|}{00.77 (95\% Cl 00.72-00.81) } & \multicolumn{2}{|l|}{00.98 (95\% Cl 00.96-00.99) } & & \\
\hline
\end{tabular}

AUC area under the curve, $L H R$ likelihood ratio, $m p M R I$ multi-parametric magnetic resonance imaging, $P V$ predictive value

Table 3 Diagnostic performance of preoperative TRUS and mpMRI for detection of extracapsular extension in the studied 58 patients

\begin{tabular}{|c|c|c|c|c|c|c|}
\hline \multirow[t]{2}{*}{ Cancer site (number) } & \multicolumn{2}{|l|}{ TRUS } & \multicolumn{2}{|l|}{ mpMRI } & \multicolumn{2}{|c|}{ Histopathology } \\
\hline & Test + ve & Test - ve & Test + ve & Test - ve & & \\
\hline & 3 & 6 & 9 & 0 & 9 & True + ve \\
\hline & 8 & 41 & 1 & 48 & 49 & True - ve \\
\hline Sensitivity (\%) & 33.33 (95\% Cl 07.49-70.07) & & 100 (95\% Cl 66.37-100) & & & \\
\hline Specificity (\%) & 83.67 (95\% Cl 70.34-92.86) & & 97.96 (95\% Cl 89.15-99.95) & & & \\
\hline Positive predictive value (\%) & 27.27 (95\% Cl 06.02-60.97) & & $90.00(95 \%$ Cl 55.50-99.75) & & & \\
\hline Negative predictive value (\%) & 87.23 (95\% Cl 74.26-95.17) & & 100 (95\% Cl 92.60-100) & & & \\
\hline Positive likelihood ratio & 02.04 (95\% Cl 00.67-06.26) & & 49.00 (95\% Cl 07.04-340.94) & & & \\
\hline Negative likelihood ratio & 00.80 (95\% Cl 00.49-01.29) & & 0.00 & & & \\
\hline AUC & 00.59 (95\% Cl 00.45-00.71) & & 00.99 (95\% Cl 00.92-1.00) & & & \\
\hline
\end{tabular}

$A U C$ area under the curve, $L H R$ likelihood ratio, $m p M R I$ multi-parametric magnetic resonance imaging, $P V$ predictive value

invasion was as follows: sensitivity (40.00\% and $100 \%)$; specificity (92.45\% and $98.11 \%)$; PPV (33.33\% and 83.33\%); and NPV (94.23\% and 100\%) (Table 4).

The ADC values were significantly higher in tumors with low grade. The apparent diffusion coefficient (ADC) values were $1 \pm 0.23 \mathrm{~mm}^{2} / \mathrm{s}$ for low-grade tumors, $\quad 0.7 \pm 0.17 \mathrm{~mm}^{2} / \mathrm{s}$ for intermediate-grade tumors and $0.5 \pm 0.13 \mathrm{~mm}^{2} / \mathrm{s}$ for high-grade tumors $(p<0.001)$.

\section{Discussion}

Currently, risk stratification and decision-making in $\mathrm{PCa}$ are largely dependent on probability tables and nomograms which are based on preoperative evaluation parameters as the serum PSA level, clinical staging by DRE and TRUS-guided prostatic biopsies. These parameters already have their pitfalls [8-10]. Therefore, there is a real need for clinicians to base therapeutic 


\begin{tabular}{lllll}
$\begin{array}{l}\text { Table } 4 \text { Diagnostic performance of preoperative } \\
\text { in the studied 58 patients }\end{array}$ & & & & \\
\hline
\end{tabular}

$A U C$ area under the curve, $L H R$ likelihood ratio, $m p M R I$ multi-parametric magnetic resonance imaging, $P V$ predictive value

decisions on not only nomograms but also advanced imaging technique.

There is a great interest in mpMRI, being a combination of the anatomical T2W imaging and functional sequences like DWI and DCE. The addition of these functional sequences which are based on special aspects of tumor cells like angiogenesis, proliferation and metabolism give the mpMRI superiority when compared with the traditional MRI that based on the anatomical sequences only $[11,12]$.

As validated by the results of the present study and most of previous studies, mpMRI has a high sensitivity and specificity in local staging and localization of PCa. With higher sensitivity and specificity, mpMRI is increasingly used in guiding biopsies in biopsy-negative and previously negative cases. Also, it is used for proper staging, risk stratification and decision-making and is being incorporated in clinical nomograms [5, 8, 9, 13-16]. Moreover, MR tractography helps visualizing the prostatic nerve bundles guiding the nerve-sparing surgery to reserve the erectile function [17].

Furthermore, there is a potential role for mpMRI in not only localizing tumor but also in identifying the areas of more aggressive cancer that could be selectively targeted by biopsy or for focal ablation therapy. Several studies have shown that both DWI and spectroscopic imaging are correlated with the Gleason grade [5, 18-20]. As confirmed by our pathologic-radiologic comparison, low $\mathrm{ADC}$ values are associated with an increasing Gleason score.

Lastly, the higher accuracy of mpMRI for diagnosis of prostate cancer makes it a proper imaging modality for identifying patients who would benefit from an active surveillance program by ruling out the presence of clinically significant disease and avoiding unnecessary prostate biopsies [16].
The major limitations of our study is the use of RP specimens as the reference standard because prostatectomy specimens are highly selected since men must be test positive for cancer on TRUS biopsy and choose to have surgery. In addition, in our study the cost-effectiveness aspect was not taken into account as the costs of mpMRI/biopsy and those of TRUS-guided biopsy were not compared.

\section{Conclusions}

The mpMRI enables the detection, localization and staging of $\mathrm{PCa}$ with reasonable sensitivity and specificity. Its combination with ultrasound should be counted on for future improvement in safety and efficacy of the prostatic biopsy procedure.

\section{Abbreviations \\ AUC: area under the curve; $\mathrm{Cl}$ : confidence interval; DCE: dynamic contrast enhancement; DRE: digital rectal examination; DWl: diffusion-weighted imag- ing; mp: multi-parametric; MRI: magnetic resonance imaging; MRS: magnetic resonance spectroscopy; NPV: negative predictive value; PCa: prostate cancer; PI-RADS: prostate imaging-reporting and data system; PPV: positive predictive value; PSA: prostatic-specific antigen; RP: radical prostatectomy; SD: standard deviation; TRUS: transrectal ultrasound.}

\section{Acknowledgements}

Not applicable.

\section{Authors' contributions}

AA contributed to data collection and analysis and manuscript writing. $\mathrm{HH}$ contributed to performing radical prostatectomies and data collection; SE contributed to protocol development and data collection; TE contributed to MRI scanning; GA contributed to histopathologic examination of specimens; MMA contributed to performing radical prostatectomies, TRUS scanning and TRUS-guided prostatic biopsies; AF contributed to protocol development and revision and edited the final version of the manuscript. All authors read and approved the final manuscript.

\section{Funding}

This study had no funding from any resource. 


\section{Availability of data and materials}

The datasets used and/or analyzed during the current study are available from the corresponding author on reasonable request.

\section{Ethics approval and consent to participate}

This study was approved by the Research Ethics Committee of the Urology Department, Al-Azhar University, Cairo, Reference Number of approval: Uro_Azhar_7_016. Informed consent: not applicable. (It is a retrospective diagnostic test accuracy study.)

\section{Consent for publication}

Not applicable. (No photographs or patients' personal information were included in this retrospective diagnostic test accuracy study.)

\section{Competing interests}

The authors declare that they have no competing interests.

\section{Author details}

1 Department of Urology, Faculty of Medicine, Al-Azhar University, Cairo 11633, Egypt. ${ }^{2}$ Department of Urology, Faculty of Medicine, Fayoum University, Fayoum, Egypt. ${ }^{3}$ Department of Radio-diagnosis, Faculty of Medicine, AlAzhar University, Cairo, Egypt. ${ }^{4}$ Department of Pathology, Faculty of Medicine, Al-Azhar University, Cairo, Egypt.

Received: 23 December 2019 Accepted: 24 February 2020

Published online: 02 July 2020

\section{References}

1. Raskolnikov D, Rais-Bahrami S, Turkbey B, Rastinehad AR, Choyke PL, Wood BJ et al (2014) Current ability of multiparametric prostate magnetic resonance imaging and targeted biopsy to improve the detection of prostate cancer. Urol Pract 1(1):13-21

2. Krahn MD, Mahoney JE, Eckman MH, Trachtenberg J, Pauker SG, Detsky AS (1994) Screening for prostate cancer: a decision analytic view. JAMA 272(10):773-780

3. Yeo L, Patel D, Bach C, Papatsoris A, Buchholz N, Junaid I et al (2011) The development of the modern prostate biopsy. In: Bissada NK (ed) Prostate Biopsy, ISBN: 978-953-307-702-4, InTech, Available from: http://www. intechopen.com/books/prostate-biopsy/thedevelopment-of-the-moder n-prostate-biopsy

4. D'Amico AV, Schnall M, Whittington R, Malkowicz SB, Schultz D, Tomaszewski JE et al (1998) Endorectal coil magnetic resonance imaging identifies locally advanced prostate cancer in select patients with clinically localized disease. Urology 51(3):449-454

5. Jansen B, Oudshoorn F, Tijans A, Yska M, Lont A, Collette E et al (2018) Local staging with multiparametric MRI in daily clinical practice: diagnostic accuracy and evaluation of a radiologic learning curve. World J Urol 36(9):1409-1415

6. Margolis DJ (2014) Multiparametric MRI for localized prostate cancer: lesion detection and staging. BioMed Res Int 2014:684127

7. Weinreb JC, Barentsz JO, Choyke PL, Cornud F, Haider MA, Macura KJ et al (2016) PI-RADS prostate imaging-reporting and data system: 2015, version 2. Eur Urol 69(1):16-40
8. Panebianco V, Barchetti F, Sciarra A, Ciardi A, Indino EL, Papalia R (eds) et al. (2015) Multiparametric magnetic resonance imaging vs. standard care in men being evaluated for prostate cancer: a randomized study. In: Urologic oncology: seminars and original investigations. Elsevier, pp. 17. e11-17. e17

9. Chun FK-H, Steuber T, Erbersdobler A, Currlin E, Walz J, Schlomm T et al (2006) Development and internal validation of a nomogram predicting the probability of prostate cancer Gleason sum upgrading between biopsy and radical prostatectomy pathology. Eur Urol 49(5):820-826

10. Ukimura O, Coleman JA, De La Taille A, Emberton M, Epstein Jl, Freedland SJ et al (2013) Contemporary role of systematic prostate biopsies: indications, techniques, and implications for patient care. Eur Urol 63(2):214-230

11. Sciarra A, Barentsz J, Bjartell A, Eastham J, Hricak H, Panebianco V et al (2011) Advances in magnetic resonance imaging: how they are changing the management of prostate cancer. Eur Urol 59(6):962-977

12. Dickinson L, Ahmed HU, Allen C, Barentsz JO, Carey B, Futterer JJ et al (2011) Magnetic resonance imaging for the detection, localisation, and characterisation of prostate cancer: recommendations from a European consensus meeting. Eur Urol 59(4):477-494

13. Panebianco V, Barchetti F, Musio D, Forte V, Pace A, De Felice F et al (2014) Metabolic atrophy and 3-T $1 \mathrm{H}$-magnetic resonance spectroscopy correlation after radiation therapy for prostate cancer. BJU Int 114(6):852-859

14. Thompson J, Lawrentschuk N, Frydenberg M, Thompson L, Stricker P, Usanz (2013) The role of magnetic resonance imaging in the diagnosis and management of prostate cancer. BJU Int 112:6-20

15. Somford DM, Hoeks CM, Hulsbergen-van de Kaa CA, HambrockT, Fütterer $J$ J, Witjes JA et al (2013) Evaluation of diffusion-weighted MR imaging at inclusion in an active surveillance protocol for low-risk prostate cancer. Invest Radiol 48(3):152-157

16. Turkbey B, Mani H, Aras O, Ho J, Hoang A, Rastinehad AR et al (2013) Prostate cancer: can multiparametric MR imaging help identify patients who are candidates for active surveillance? Radiology 268(1):144-152

17. Panebianco V, Barchetti F, Sciarra A, Marcantonio A, Zini C, Salciccia S et al (2013) In vivo 3D neuroanatomical evaluation of periprostatic nerve plexus with 3T-MR diffusion tensor imaging. Eur J Radiol 82(10):1677-1682

18. Claus FG, Hricak H, Hattery RR (2004) Pretreatment evaluation of prostate cancer: role of MR imaging and $1 \mathrm{H}$ MR spectroscopy. Radiographics 24(suppl_1):S167-S180

19. Kobus T, Vos PC, HambrockT, De Rooij M, Hulsbergen-Van de Kaa CA, Barentsz JO et al (2012) Prostate cancer aggressiveness: in vivo assessment of MR spectroscopy and diffusion-weighted imaging at 3 T. Radiology 265(2):457-467

20. Hambrock T, Hoeks C, Hulsbergen-van de Kaa C, Scheenen T, Fütterer J, Bouwense S et al (2012) Prospective assessment of prostate cancer aggressiveness using 3-T diffusion-weighted magnetic resonance imaging-guided biopsies versus a systematic 10-core transrectal ultrasound prostate biopsy cohort. Eur Urol 61(1):177-184

\section{Publisher's Note}

Springer Nature remains neutral with regard to jurisdictional claims in published maps and institutional affiliations. 